MATEC Web of Conferences 51, 01008 (2016)

DOI: $10.1051 /$ matecconf/20165101008

C) Owned by the authors, published by EDP Sciences, 2016

\title{
A Multi-Leveled Coordinate for Multibody Mechanics
}

\author{
Fan Shen ${ }^{1}$, Siyuan Rong ${ }^{1, a}$, Jiafu Liư ${ }^{2}$, Naigang Cui ${ }^{1}$ \\ ${ }^{1}$ Department of Space Engineering, Harbin Institute of Technology, Harbin, Heilongjiang, China \\ ${ }^{2}$ College of Aerospace Engineering, Shenyang Aerospace University, Shenyang, Liaoning China
}

\begin{abstract}
For a proper way of modelling and simulation, as well as the precise and completeness, and a new multi-level method named homogeneous tensor coordinate(HTC) was developed by improving the absolute nodal coordinate formulation(ANCF) and the homogeneous coordinates(HC). In HTC, a new form of $2 \times 2$ matrix was employed instead of angles, when the capacity of expression and calculation was increased at the same time. HTC has good performance in traditional rigid body mechanics, which especially suitable for multibody modelling. Therefore, the robotic engineering may benefit from this finding, and a new formulation for scientific research may also affect the physics although some mathematical problems are raised in matrix theory.
\end{abstract}

\section{Introduction}

As a cornerstone of modern science, classical mechanics was a mature and reliable theory[1]. However, the accuracy of classical mechanics is not able to meet the increasing demands of modern research or engineering. As a representative of the analytical modelling, floating frame method does not have a sufficiently general form and accuracy for questions as highly-flexible problem for the angle components[2]. And for some particularly difficult problems there is even not a unified theory, for that there is various limited models for turbulence. The accuracy and completeness can be concluded to a basic question in science: how to describe an object in proper approximation. Multiorder method could be one of the answers, which means, use a specific value in a specific scale.

The inaccuracy may be caused by the incompleteness. The problem will be confusion while using a basic tool, but may be simple if we use a complicated modelling method. It can be a selectable way to solve a question precisely by changing our basic theories.

In most differential equation, multi-orders are used to describe the characteristics of different objects in various scale. And in the history of maths, physics and engineering, there are many expressions composed with multiple components, such as plural and quaternion[3], which use linear superposition of the base band to represent, when vector[4] and tensor[5] are represented by a matrix form. The control equation[2] is also a common form of multi-leveled applications in particular, which is not composed with multiple dimension, but with different orders of a single dimension. Modern control theory constituted in the equations above, and reached a high maturity not only in forms but also in theory. Taylor polynomial expansion[6] was always used in computation with nonlinear or uncertain values, which has different substrates and will represents approximated value more accurately.

\footnotetext{
${ }^{a}$ Corresponding author : rage2004@163.com
} 
More and more approximated linear formulations were derived and used in multi-orders, for that there is enough theoretical basis and application environments already. Therefore, an advanced maths method for describing multiple levels was proposed. Meanwhile, a new coordinate in matrix form was raised for the practice of this method by referring the Homogeneous coordinates (HC)[7] and Absolute Coordinate Nodal Formulation (ANCF)[5].

ANCF is a method for FEM raised by Prof A.A.Shabana[2,8,9], which uses all the first and second order component values of a node in 3 -D coordinate. It abandoned the classical angle component $\theta$, because the angles are actually functions resulted by $\mathrm{x}, \mathrm{y}, \mathrm{z}$ in nature. ANCF is an accurate and complete method which uses 12 components to describe a node, and has solved many practical problems $[10,11,6]$. However, the length of array and the unusual rotational description made it difficult to describe the coordinate, there should be a more simple form instead.

$\mathrm{HC}$ is an excellent tool with completeness August Ferdinand Möbius created[12], which could use matrix multiplication instead of vector. Due to the special definition and operations, it is not widely used. However, its idea was inherited by many other methods. Such as discrete time transfer matrix method, Xiaoting RUI uses linearized transfer matrix for multibody system dynamics, although the result still have the problem of premature linearized and was not satisfied enough.

Thus, to merge the advantages in HC and ANCF, an improved coordinate is represented in this paper. The new method uses the absolute precise tensor components in ANCF and abandoned the angle description, when a two-dimensional matrix is constructed according to the idea of HC, for easier derivation and operation. The method was successfully applied in rigid body mechanics, which has a more complete and concise physical description and a more accurate mathematical form.

\section{A Multi-Levelled Expression}

To give out a multi-leveled expression, we need an array could be written as

$$
S=\left\{s, \frac{\partial s}{\partial x}, \frac{\partial s}{\partial y}, \cdots, \frac{\partial^{2} s}{\partial x^{2}}, \frac{\partial^{2} s}{\partial x \partial y}, \frac{\partial^{2} s}{\partial y^{2}}, \cdots\right\}
$$

There is a gradient operator

$$
\nabla=\left(\begin{array}{lll}
\frac{\partial}{\partial x} & \frac{\partial}{\partial y} & \frac{\partial}{\partial z}
\end{array}\right)
$$

Then we define a potential function $\phi$, and $\nabla \phi=(\underline{x}, \underline{y}, \underline{z})$, according to the homogeneous coordinates, set the zero-order value to be 1 , we obtain

$$
S=\{1, \nabla \phi, \nabla \nabla \phi, \nabla \nabla \nabla \phi, \cdots\}
$$

The complete multi-levelled equation should be

$$
f\left(S_{1}, S_{2}, \cdots\right)=0
$$

For not all orders could be considered, we need to determine a appropriate number of orders to use. Particular problems and mathematical algorithms should be examined to give out the answer.

Although the operation of this method is unclear, for simple operation, its significance is obvious. In basis i, we have $S_{i}^{j}=\nabla_{i} \phi_{j}, S_{i i}^{j}=\nabla_{i} \nabla_{i} \phi_{j}$, then the complete form should be

$$
S_{i}^{j}=\left\{1, s_{i}^{j}, s_{i i}^{j}, \cdots\right\}
$$

$S(n)$ is the $n$ th-order component in $\mathrm{S}$, such as $S(2)=S_{i j}$, according to the algorithm of tensor, easily we have

$$
\begin{gathered}
S_{i}^{j}(n) S_{j}^{k}(0)=S_{i}^{j}(n),(n=1,2,3, \cdots) \\
S_{i}^{j}(n+1) S_{j}^{k}(1)=S_{i}^{j}(n),(n=1,2,3, \cdots)
\end{gathered}
$$




$$
S_{i}^{j}(n+2) S_{j}^{k}(2)=S_{i i}^{k}(n),(n=1,2,3, \cdots)
$$

Transfer rules for any order could be derived from the algorithm of tensor, but for higher order there is no clear definition and value to use out of equation(6), (7) and (8), deeper theory will be discussed in further study.

\section{The Homogeneous Tensor Coordinate}

A brief form is needed as the ideal form had been given, so we take the order 0,1 and 2 for construction.

A space Cartesian coordinate system was defined by $x_{i} \quad y_{i} z_{i}$ with the subscribe $\mathrm{i}$, consider the potential function and gradient operator, with the tensor-like expression, the matrix could be defined as

$$
\mathbf{T}_{i}^{j}=\nabla_{i} \phi_{j}=\left(\begin{array}{l}
\frac{\partial \phi_{j}}{\partial x_{i}} \\
\frac{\partial \phi_{j}}{\partial y_{i}} \\
\frac{\partial \phi_{j}}{\partial z_{i}}
\end{array}\right), \boldsymbol{T}_{i i}^{j}=\nabla_{i} \nabla_{i}^{T} \phi_{j}=\left(\begin{array}{lll}
\frac{\partial^{2} \phi_{j}}{\partial x_{i} \partial x_{i}} & \frac{\partial^{2} \phi_{j}}{\partial x_{i} \partial y_{i}} & \frac{\partial^{2} \phi_{j}}{\partial x_{i} \partial z_{i}} \\
\frac{\partial^{2} \phi_{j}}{\partial y_{i} \partial x_{i}} & \frac{\partial^{2} \phi_{j}}{\partial y_{i} \partial y_{i}} & \frac{\partial^{2} \phi_{j}}{\partial y_{i} \partial z_{i}} \\
\frac{\partial^{2} \phi_{j}}{\partial z_{i} \partial x_{i}} & \frac{\partial^{2} \phi_{j}}{\partial z_{i} \partial y_{i}} & \frac{\partial^{2} \phi_{j}}{\partial z_{i} \partial z_{i}}
\end{array}\right)
$$

Combine 3 matrix above, appropriately, then we have a $4 \times 4$ matrix. With the gradient symbol, we defined operator $\mathrm{L}_{i}$ as

$$
\mathrm{L}_{i}=\left(\begin{array}{cc}
1 & 0 \\
\nabla_{i} & \nabla_{i} \nabla_{i}^{T}
\end{array}\right)
$$

This is an extension of homogeneous coordinates. Let the potential function to be 1, which places at its upper left corner, then the addition of matrix is changed to multiplication like $\mathrm{HC}$, with a series of similar expression and computational advantages. There are 12 remaining non-zero value, which correspond to the components of ANCF, with a second-order accuracy in one node.

With respect to the authors of ANCF and $\mathrm{HC}$, in this paper we call it homogeneous tensor coordinate(HTC), whose operations and meanings will be given later in the paper.

HTC follow all the matrix algorithms with a series of operational advantages. Defining $\mathbf{R}_{i}^{j}=\mathrm{L}_{i} \phi_{j}$, the algorithms is presented as below.

1. The addition and subtraction of HTC. When the matrix have the same subscript it is easy to known by block matrix algorithms that

$$
\mathbf{R}_{i}^{j} \pm \mathbf{R}_{i}^{k}=\left(\begin{array}{cc}
\mathbf{T}^{j} & 0 \\
\mathbf{T}_{i}^{j} & \mathbf{T}_{i i}^{j}
\end{array}\right)+\left(\begin{array}{cc}
\mathbf{T}^{k} & 0 \\
\mathbf{T}_{i}^{k} & \mathbf{T}_{i i}^{k}
\end{array}\right)=\left(\begin{array}{cc}
\mathbf{T}^{j} \pm \mathbf{T}^{k} & 0 \\
\mathbf{T}_{i}^{j} \pm \mathbf{T}_{i}^{k} & \mathbf{T}_{i i}^{j} \pm \mathbf{T}_{i i}^{k}
\end{array}\right)
$$

Obviously, these two operations are allowed, although the significance of addition remains uncertain when $T^{i}$ and $T^{k}$ are all non-zero.

2.The multiplication and division of HTC. Since the HTC is the matrix form, it will be directionality. Consulting the vector multiplication can be divided into ordinary multiplication, dot product and cross product. Multiplication operate could be 


$$
\begin{gathered}
\mathbf{R}_{i}^{j} \mathbf{R}_{j}^{k}=\left(\begin{array}{cc}
\mathbf{T}^{j} & 0 \\
\mathbf{T}_{i}^{j} & \mathbf{T}_{i i}^{j}
\end{array}\right)\left(\begin{array}{cc}
\mathbf{T}^{k} & 0 \\
\mathbf{T}_{i}^{k} & \mathbf{T}_{i i}^{k}
\end{array}\right)=\left(\begin{array}{cc}
\mathbf{T}^{j} \mathbf{T}^{k} & 0 \\
\mathbf{T}_{i}^{j} \mathbf{T}^{k}+\mathbf{T}_{i i}^{j} \mathbf{T}_{j}^{k} & \mathbf{T}_{i i}^{j} \mathbf{T}_{j j}^{k}
\end{array}\right) \\
\mathbf{R}_{i}^{j} \cdot \mathbf{R}_{i}^{k}=\mathbf{R}_{i}^{j T} \mathbf{R}_{i}^{k}=\left(\begin{array}{cc}
\mathbf{T}^{j T} & \mathbf{T}_{i}^{j T} \\
0 & \mathbf{T}_{i i}^{j T}
\end{array}\right)\left(\begin{array}{cc}
\mathbf{T}^{k} & 0 \\
\mathbf{T}_{i}^{k} & \mathbf{T}_{i i}^{k}
\end{array}\right)=\left(\begin{array}{cc}
\mathbf{T}^{j T} \mathbf{T}^{k}+\mathbf{T}_{i}^{j} \cdot \mathbf{T}_{i}^{k} & \mathbf{T}_{i}^{j T} \mathbf{T}_{i i}^{k} \\
\mathbf{T}_{i i}^{j T} \mathbf{T}_{j}^{k} & \mathbf{T}_{i i}^{j T} \mathbf{T}_{j j}^{k}
\end{array}\right)
\end{gathered}
$$

Cross product is only for an array, so it can be

$$
\mathbf{R}_{i}^{j} \times \mathbf{R}_{i}^{k}=\left(\begin{array}{cc}
1 & 0 \\
\mathbf{T}_{i}^{j} & 0
\end{array}\right) \times\left(\begin{array}{cc}
1 & 0 \\
\mathbf{T}_{i}^{j} & 0
\end{array}\right)=\left(\begin{array}{cc}
1 & 0 \\
0 & \tilde{\mathbf{T}}_{i}^{j}
\end{array}\right)\left(\begin{array}{cc}
1 & 0 \\
\mathbf{T}_{i}^{j} & 0
\end{array}\right)
$$

where

$$
\tilde{\mathbf{T}}_{i}^{j} T_{i}^{k}=\mathbf{T}_{i}^{j} \times \mathbf{T}_{i}^{k}
$$

Division is the inverse operation of multiplication, which can consult the block matrix.

3.The differential and Integral of HTC. According to the differential law of matrix and tensor, we could differentiate each element in the HTC when the matrix needs to be differentiated.

$$
\frac{\partial \mathbf{R}_{i}^{j}}{\partial t}=\left(\begin{array}{cc}
\frac{\partial \mathbf{T}^{j}}{\partial t} & 0 \\
\frac{\partial \mathbf{T}_{i}^{j}}{\partial t} & \frac{\partial \mathbf{T}_{i i}^{j}}{\partial t}
\end{array}\right)
$$

We can see from the paragraph that HTC can be used to represent a multi-levelled information, whose low-level can provide an easy form for the theoretical equation, when the high-level provides a sufficient high-precision. This indicated would give rise to two problems: 1 . Commonly used firstorder vector become a second-order matrix, third-order operation may also appear in using HTC, when there is still no higher-dimensional matrix theory yet. a new requirements of matrix analysis theory is put forward. 2. It is predictable that a coupling effect will occur in the state variables which will be 2-D matrix instead of 1-D array. It is also a problem had never be dealt with.

However, we have to face these problems rather than to avoid. Compared to the classic expression, this multi-levelled expression is more in line with the objective world. Since the invention of calculus, it is the first time a clear representation defined for the hierarchical data. It is worth using such a matrix for the need of more accurate research. And in nature, there is no variable non-independent, the additional equation to keep the balance between translational and rotational movement may be pass off with the proposal of HTC.

\section{HTC for Rigid Body Mechanics}

\subsection{Expression in mathematics}

\subsubsection{Basic form}

Let $x_{i}=\frac{\partial \phi}{\partial x}$, the coordinate for a node could be written as

$$
\mathbf{R}_{o}^{i}=\left(\begin{array}{cc}
1 & 0 \\
\mathbf{x}_{o}^{i} & \boldsymbol{\theta}_{o}^{i}
\end{array}\right)
$$

where 


$$
\mathbf{x}_{o}^{i}=\left(\begin{array}{c}
x_{i} \\
y_{i} \\
z_{i}
\end{array}\right), \quad \boldsymbol{\theta}_{o}^{i}=\left(\begin{array}{lll}
\frac{\partial x_{i}}{\partial x_{o}} & \frac{\partial x_{i}}{\partial y_{o}} & \frac{\partial x_{i}}{\partial z_{o}} \\
\frac{\partial y_{i}}{\partial x_{o}} & \frac{\partial y_{i}}{\partial y_{o}} & \frac{\partial y_{i}}{\partial z_{o}} \\
\frac{\partial z_{i}}{\partial x_{o}} & \frac{\partial z_{i}}{\partial y_{o}} & \frac{\partial z_{i}}{\partial z_{o}}
\end{array}\right)
$$

According to the definition and algorithms of HC, the translational matrix can be written as $\mathbf{X}_{o}^{i}$. Similarly, consulting the definition of ANCF, the angle definition can be given as $\boldsymbol{\Theta}_{o}^{i}$

$$
\mathbf{X}_{o}^{i}=\left(\begin{array}{cc}
1 & 0 \\
\mathbf{x}_{o}^{i} & \mathbf{I}
\end{array}\right), \quad \boldsymbol{\Theta}_{o}^{i}=\left(\begin{array}{cc}
1 & 0 \\
\mathbf{0} & \boldsymbol{\theta}_{o}^{i}
\end{array}\right)
$$

\subsubsection{Equivalence for small deformation}

When only small spatial displacement occur, We can decompose the matrix into $\mathbf{R}_{o}^{i}=\hat{\mathbf{R}}_{o}^{i}+\tilde{\mathbf{R}}_{o}^{i}$ as

$$
\hat{\mathbf{R}}_{o}^{i}=\left(\begin{array}{cc}
1 & 0 \\
\mathbf{0} & \hat{\boldsymbol{\theta}}_{o}^{i}
\end{array}\right), \quad \tilde{\mathbf{R}}_{o}^{i}=\left(\begin{array}{cc}
1 & 0 \\
\mathbf{x}_{i} & \tilde{\boldsymbol{\theta}}_{o}^{i}
\end{array}\right)
$$

where

$$
\begin{gathered}
\hat{\boldsymbol{\theta}}_{o}^{i}=\left(\begin{array}{ccc}
\frac{\partial x_{i}}{\partial x_{o}} & \frac{1}{2}\left(\frac{\partial x_{i}}{\partial y_{o}}+\frac{\partial y_{i}}{\partial x_{o}}\right) & \frac{1}{2}\left(\frac{\partial x_{i}}{\partial z_{o}}+\frac{\partial z_{i}}{\partial x_{o}}\right) \\
\frac{1}{2}\left(\frac{\partial y_{i}}{\partial x_{o}}+\frac{\partial x_{i}}{\partial y_{o}}\right) & \frac{\partial y_{i}}{\partial y_{o}} & \frac{1}{2}\left(\frac{\partial y_{i}}{\partial z_{o}}+\frac{\partial z_{i}}{\partial y_{o}}\right) \\
\frac{1}{2}\left(\frac{\partial x_{i}}{\partial z_{o}}+\frac{\partial z_{i}}{\partial x_{o}}\right) & \frac{1}{2}\left(\frac{\partial y_{i}}{\partial z_{o}}+\frac{\partial z_{i}}{\partial y_{o}}\right) & \frac{\partial z_{i}}{\partial z_{o}}
\end{array}\right) \\
\tilde{\boldsymbol{\theta}}_{o}^{i}=\left(\begin{array}{ccc}
0 & \frac{1}{2}\left(\frac{\partial x_{i}}{\partial y_{o}}-\frac{\partial y_{i}}{\partial x_{o}}\right) & \frac{1}{2}\left(\frac{\partial x_{i}}{\partial z_{o}}-\frac{\partial z_{i}}{\partial x_{o}}\right) \\
\frac{1}{2}\left(\frac{\partial y_{i}}{\partial x_{o}}-\frac{\partial x_{i}}{\partial y_{o}}\right) & 0 & \frac{1}{2}\left(\frac{\partial y_{i}}{\partial z_{o}}-\frac{\partial z_{i}}{\partial y_{o}}\right) \\
\frac{1}{2}\left(\frac{\partial x_{i}}{\partial z_{o}}-\frac{\partial z_{i}}{\partial x_{o}}\right) & \frac{1}{2}\left(\frac{\partial y_{i}}{\partial z_{o}}-\frac{\partial z_{i}}{\partial y_{o}}\right) & 0
\end{array}\right)
\end{gathered}
$$

$\hat{\mathbf{R}}_{o}^{i}$ is a matrix for deformation in elastic mechanics, which does not exist in rigid body movement, therefore it is 0 . $\tilde{\mathbf{R}}_{o}^{i}$ can be written in Euler angles form approximatively as

$$
\tilde{\mathbf{R}}_{o}^{i}=\left(\begin{array}{cccc}
1 & 0 & 0 & 0 \\
x_{i} & 0 & -\psi_{i} & \vartheta_{i} \\
y_{i} & \psi_{i} & 0 & -\theta_{i} \\
z_{i} & -\vartheta_{i} & \theta_{i} & 0
\end{array}\right)
$$


Equation (24) is composed with translational matrix and angle matrix. The node make the translational movement first, then finish the rotational movement. Two movement constitute a coordinate expression, so we have $\tilde{\mathbf{R}}_{o}^{j}=\tilde{\mathbf{X}}_{i}^{j} \tilde{\boldsymbol{\Theta}}_{o}^{i}$, where $\tilde{\boldsymbol{\Theta}}_{o}^{i}$ is a translational movement, when $\tilde{\boldsymbol{\Theta}}_{o}^{i}$ is for rotating. Note that $\tilde{\mathbf{R}}_{o}^{j} \neq \tilde{\boldsymbol{\Theta}}_{o}^{j} \tilde{\mathbf{X}}_{i}^{j}$.

\subsubsection{Equivalence for large deformation}

Considering the normal condition, we use Euler angle for example, according to the order $x(\alpha) \rightarrow y(\beta) \rightarrow x(\gamma)$, and write $\mathrm{s} \beta=\sin \beta$ and $\mathrm{c} \theta=\cos \theta$, a successful transformation in HTC could be

$$
\overline{\mathbf{R}}_{o}^{i}=\mathbf{C}_{x}(\gamma) \mathbf{C}_{z}(\beta) \mathbf{C}_{x}(\alpha) \mathbf{R}_{o}^{i}
$$

where

$$
\mathbf{C}_{x}(\gamma)=\left(\begin{array}{cccc}
1 & 0 & 0 & 0 \\
0 & 1 & 0 & 0 \\
0 & 0 & \mathrm{c} \gamma & -\mathrm{s} \gamma \\
0 & 0 & \mathrm{~s} \gamma & \mathrm{c} \gamma
\end{array}\right), \quad \mathbf{C}_{z}(\beta)=\left(\begin{array}{cccc}
1 & 0 & 0 & 0 \\
0 & \mathrm{c} \beta & 0 & \mathrm{~s} \beta \\
0 & 0 & 1 & 0 \\
0 & -\mathrm{s} \beta & 0 & \mathrm{c} \beta
\end{array}\right)
$$

The translation matrix (25) kept the translational and rotational displacement in one matrix, which has an operational result consistent with classical mechanics theory.

\subsection{The operation and meaning in mechanics}

As the coordinate defined, we will then derive its algorithm.

\subsubsection{Minus and multiplication}

We can use the subtraction between $\mathbf{R}_{o}^{i}$ and $\mathbf{R}_{o}^{j}$ to represent the displacement of nodes. In HTC form it can be written as

$$
\overline{\mathbf{R}}_{o}^{i, j}=\mathbf{R}_{o}^{j}-\mathbf{R}_{o}^{i}
$$

The transfer of coordinates can be achieved with the HTC matrix multiplication. Using subscript as the basis of coordinate, superscript as the symbol, according to the algorithm of the matrix and tensor, it is easy to prove

$$
\mathbf{R}_{o}^{j}=\mathbf{R}_{o}^{i} \mathbf{R}_{i}^{j}
$$

\subsubsection{Intergrate and difference for order changing}

For the differential form of HTC, differentiate equation will be

$$
\partial \dot{\mathbf{R}}_{o}^{i}=\frac{\partial \mathbf{R}_{o}^{i}}{\partial t}
$$

According to the matrix algorithms, the homogeneous item become 0 after differentiation, which means it is no longer a displacement in physical meaning, but a velocity coordinate instead. If we differentiate the equation with the time variable $\mathrm{t}$, then we have

$$
\partial \dot{\mathbf{R}}_{o}^{k}=\partial \dot{\mathbf{R}}_{o}^{i} \mathbf{R}_{i}^{j} \mathbf{R}_{j}^{k}+\partial \mathbf{R}_{o}^{i} \dot{\mathbf{R}}_{i}^{j} \mathbf{R}_{j}^{k}+\partial \mathbf{R}_{o}^{i} \mathbf{R}_{i}^{j} \dot{\mathbf{R}}_{j}^{k}
$$


We could learn from equation that the homogeneous item will be 0 in the sum of three matrix, that is, the HTC matrix is a description for speed now. Meanwhile, according to the physical meaning of rigid body motion, if the $R_{o}^{i}, R_{i}^{j}, R_{j}^{k}$ were kinematic pairs, the 3 items in addition corresponds to convected speed caused by 3 pairs, while the result is its real speed of body.

However, this represents have some shortcomings: 1. The homogeneous item of acceleration differentiated by velocity is the same as velocity in HTC, which will be 0 and may bring some confusion in description. 2 . Having the value 0 , homogeneous item in velocity and acceleration can not be integrated to displacement which has value 1 there. Strict mathematical definition needs further study.

\subsubsection{Dot-multiply for kinetic and potential energy}

For classical mechanics, kinetic and potential energy is needed. According to kinetic equation in vector coordinates and the dot product operation in HTC defined, the kinetic energy for HTC can be written as

$$
\mathbf{E}_{V}=\frac{1}{2} m \dot{\mathbf{R}}^{T} \dot{\mathbf{R}}
$$

For rigid coordinates, we define $\dot{\mathbf{R}}_{a}=\dot{\boldsymbol{\Theta}}_{a} \mathbf{X}_{a}+\boldsymbol{\Theta}_{a} \dot{\mathbf{X}}_{a}$, then we have

$$
\mathbf{E}_{V}=\frac{1}{2} m\left(\mathbf{X}_{a}^{T} \dot{\Theta}_{a}^{T}+\dot{\mathbf{X}}_{a}^{T} \Theta_{a}^{T}\right)\left(\dot{\Theta}_{a} X+\mathbf{\Theta}_{a} \dot{\mathbf{X}}_{a}\right)=\mathbf{E}_{X_{a} X_{a}}+\mathbf{E}_{\Theta_{a} \Theta_{a}}+\mathbf{E}_{X_{a} \Theta_{a}}+\mathbf{E}_{\Theta_{a} X_{a}}
$$

where

$$
\left\{\begin{array}{l}
\mathbf{E}_{\mathbf{X}_{a} \mathbf{X}_{a}}=\frac{1}{2} m \dot{\mathbf{X}}_{a}^{T} \boldsymbol{\Theta}_{a}^{T} \boldsymbol{\Theta}_{a} \dot{\mathbf{X}}_{a} \\
\mathbf{E}_{\mathbf{X}_{a} \boldsymbol{\Theta}_{a}}=\frac{1}{2} m \dot{\mathbf{X}}_{a}^{T} \boldsymbol{\Theta}_{a}^{T} \dot{\boldsymbol{\Theta}}_{a} \mathbf{X}_{a}
\end{array},\left\{\begin{array}{l}
\mathbf{E}_{\boldsymbol{\Theta}_{a} \boldsymbol{\Theta}_{a}}=\frac{1}{2} m \mathbf{X}_{a}^{T} \dot{\boldsymbol{\Theta}}_{a}^{T} \dot{\boldsymbol{\Theta}}_{a} \mathbf{X}_{a} \\
\mathbf{E}_{\boldsymbol{\Theta}_{a} \mathbf{x}_{a}}=\frac{1}{2} m \mathbf{X}_{a}^{T} \dot{\boldsymbol{\Theta}}_{a}^{T} \boldsymbol{\Theta}_{a} \dot{\mathbf{X}}_{a}
\end{array}\right.\right.
$$

The $\mathbf{E}_{\mathbf{x}_{a} \mathbf{x}_{a}}$ is the translational kinetic energy, when $\mathbf{E}_{\mathbf{\Theta}_{a} \boldsymbol{\Theta}_{a}}$ is the pure rotational kinetic energy. The remaining two item $\mathbf{E}_{\mathbf{X}_{a} \boldsymbol{\Theta}_{a}} \mathbf{E}_{\Theta_{a} \mathbf{x}_{a}}$ is the coupled kinetic energy. Especially, for a rotational energy, if there is $\mathbf{X}_{b}$, and

$$
\mathbf{E}_{V}=\frac{1}{2} m \mathbf{X}_{a}^{T} \dot{\boldsymbol{\Theta}}_{a}^{T} \mathbf{X}_{b}^{T} \mathbf{X}_{b} \dot{\boldsymbol{\Theta}}_{a} \mathbf{X}_{a}
$$

The $\mathbf{X}_{b}^{T} \mathbf{X}_{b}$ will consist the Parallel Axis Theorem, when $\mathrm{m}, \mathbf{X}_{a}^{T}, \mathbf{X}_{a}$ consist the moment of inertia. The results are consistent with the classical equations.

\subsection{Summary}

From this section we can conclude that HTC has a perfect description for solid body mechanics. The multi-levelled and tensor-formed values can be described in one HTC. In case that the node have a physical meaning, the HTC follow the general operating rules of matrix.

Therefore we can learn from this paper that: the angle may be the root of many problems such as nonlinearity when the rotational description are used in the same level together with translational description. If we improve this to the HTC description, more accurate answers may come true in the future research. That may be a help for highly-flexible mechanics and so on.

However, there is also some problem raised as well as new directions provided: 1 . Multi-levelled description needs strict definition and demonstrate in mathematics. 2. The matrix may have to be 
extended to more than 2 dimensions, in order to accommodate the higher operational needs, which need new matrix analysis theory to accomplish.

\section{Conclusion}

A level-based mathematical expression and algorithm was raised, a new tensor-formed matrix called HTC was defined and the operating rule was also given by referring the ANCF and HC, which agrees well with the current theory in rigid body mechanics. This method provides some innovation for related subjects, although there is still many theory need to develop.

The article made some arrangements in classical theory of Newtonian mechanics. Based on the multi-level thought, HTC can be gradually improved in follow-up works, modelling and simulation may become more comfortable especially in multibody mechanics. The usage of HTC in other object is still unclear and more development is needed.

\section{Acknowledgement}

This work was supported partially by the National Natural Science Foundation of China (Project Number: 11272101, 11302134), Research Fund for Doctoral Program of Higher Education of China (Project Number: 20102302110051), the 12th Five-Year Plan Projects for civil aerospace of China, the innovation fund of China aerospace science and technology group.

\section{References}

1. I. Newton, Mathematical principles of natural philosophy. A. Strahan (1802)

2. A. A. Shabana and T. Note, Definition of the slopes and the finite element absolute nodal coordinate formulation, Multibody System Dynamics, 1(3), pp. 339-348 (1997)

3. P. G. Tait, An elementary treatise on quaternions. University Press (1890)

4. A. A. Shabana, Flexible Multibody Dynamics: Review of Past and Recent Developments, Multibody System Dynamics, 1, 189-222, June (1997)

5. A. A. Shabana, H. A. Hussien, and J. L. Escalona, Application of the absolute nodal coordinate formulation to large rotation and large deformation problems, Journal of Mechanical Design, 120(2), 188-195 (1998)

6. A. A. Shabana and R. Y. Yakoub, Three Dimensional Absolute Nodal Coordinate Formulation for Beam Elements: Theory, Journal of Mechanical Design, 123(4), 606-613, 2000.

7. L. E. Garner, An outline of projective geometry. North Holland New York and Oxford (1981)

8. G. G. Sanborn and A. A. Shabana, A rational finite element method based on the absolute nodal coordinate formulation, Nonlinear Dynamics, 58(3), 565-572 (2009)

9. G. G. Sanborn and A. A. Shabana, On the integration of computer aided design and analysis using the finite element absolute nodal coordinate formulation, Multibody System Dynamics, 22(2), 181-197 (2009)

10. H. Sugiyama and A. A. Shabana, Application of plasticity theory and absolute nodal coordinate formulation to flexible multibody system dynamics, Journal of Mechanical Design, 126(3), pp. 478-487 (2004)

11. R. Y. Yakoub and A. A. Shabana, Three Dimensional Absolute Nodal Coordinate Formulation for Beam Elements: Implementation and Applications, Journal of Mechanical Design, 123(4), 614-621, (1999)

12. F. Berchtold and J. Hausen, Homogeneous coordinates for algebraic varieties, Journal of Algebra, 266(2), 636-670 (2003) 